\title{
Phase 0 Trial
}

National Cancer Institute

\section{Source}

National Cancer Institute. Phase O Trial. NCI Thesaurus. Code C54721.

A clinical trial that uses an investig ational agent that is available only in very limited quantities and which has never previously given to humans or for which there is extremely limited human experience. Phase 0 clinical trials are intended to enable researchers to understand the path of the drug in the body and its efficacy. Adverse event reporting in Phase 0 trials is expedited. 